\title{
ARTIGOS
}

\section{PROBLEMÁTICAS E DESAFIOS DA PESQUISA QUALITATIVA NA EDUCAÇÃO BRASILEIRA SOB O INESQUECÍVEL OLHAR DE MARLI ANDRÉ}

MARTINS, Pura Lúcia Oliver Pontificia Universidade Católica do Paraná - PUCPR

Curitiba, Paraná pura.oliver@pucpr.br https://orcid.org/0000-0003-1639-0798

PINTO, Neuza Bertoni Rede Amazônica de Educação em Ciências e Matemática - REAMEC Universidade Federal de Mato Grosso- UFMT

Cuiabá, Mato Grosso neuzabertonip@gmail.com https://orcid.org/000-0002-9224-3020

RESUMO: O nome de Marli André consagrou-se no cenário educacional brasileiro como grande referência das pesquisas qualitativas. Durante mais de cinco décadas, a pesquisadora não perdeu de vista as problemáticas e os desafios que permearam as pesquisas dessa vertente metodológica. Para melhor compreender o expressivo legado deixado por ela às diferentes gerações de pesquisadores que buscam fundamentar teórica e metodologicamente sua produção científica, este texto vale-se de um conjunto de artigos selecionados, publicados em diferentes periódicos nacionais. Situar, nos caminhos percorridos por André, questionamentos e avanços na produção de conhecimentos sobre a pesquisa qualitativa, possibilitou analisar os inúmeros desafios que a pesquisadora destacou em sua trajetória, especialmente aqueles decorrentes das dúvidas e soluções identificadas na formação de pesquisadores da área da educação.

PALAVRAS-CHAVE: Marli André. Pesquisa Qualitativa. Problemáticas. Desafios. Formação de Pesquisadores. 


\title{
PROBLEMS AND CHALLENGES OF QUALITATIVE RESEARCH IN BRAZILIAN EDUCATION UNDER THE UNFORGETTABLE LOOK OF MARLI ANDRÉ
}

\begin{abstract}
The name of Marli André was consecrated itself in the Brazilian educational scene as a great reference of qualitative research. For more than five decades, the researcher did not lose sight of the problems and challenges that permeated the research from this methodological point of view. To better understand the expressive legacy left by her to the different generations of researchers who seek to theoretically and methodologically support their scientific production, this text is based on a set of selected articles, published in different national journals. To situate, along the paths taken by André, questions and advances in the production of knowledge about qualitative research, made it possible to analyze the numerous challenges that the researcher highlighted in her trajectory, especially those arising from the doubts and solutions identified in the researchers qualification in the area of education.
\end{abstract}

KEYWORDS: Marli André. Qualitative Research. Problematic. Défis. Researchers Qualification

\section{PROBLÉMATIQUES ET DÉFIS DE LA RECHERCHE QUALITATIVE DANS L'ÉDUCATION BRÉSILIENNE SOUS LE REGARD INOUBLIABLE DE MARLI ANDRÉ}

RÉSUMÉ: Le nom de Marli André a été consacré sur la scène éducative brésilienne comme une grande référence en recherche qualitative. Depuis plus de cinq décennies, le chercheur n'a pas perdu de vue les problématiques et les défis qui ont imprégné la recherche dans cet aspect méthodologique. Afin de mieux comprendre l'héritage expressif qu'il a laissé aux différentes générations de chercheurs qui cherchent à soutenir théoriquement et méthodologiquement leur production scientifique, ce texte s'appuie sur un ensemble d'articles sélectionnés, publiés dans différentes revues nationales. Situer, dans les chemins empruntés par la chercheuse, les questions et les avancées dans la production de connaissances sur la recherche qualitative, a permis d'analyser les nombreux défis que la chercheuse a mis en évidence dans sa trajectoire, notamment ceux découlant des doutes et des solutions identifiés dans la formation de chercheurs dans le domaine de l'éducation.

MOTS-CLÉS: Marli André. Recherche qualitative. Problématiques. Avances. Formation des chercheurs. 
DOI https://doi.org/10.31639/rbpfp.v13i28.543

\section{Introdução}

A pesquisa qualitativa em educação no Brasil tem como grande referência Marli André que durante mais de cinco décadas dedicou-se a estudar as problemáticas e os desafios que permearam as pesquisas dessa vertente metodológica, sempre buscando resgatar a trajetória da pesquisa na área de educação para melhor compreender suas lacunas e possibilidades de avanços.

A historicização da pesquisa feita por Marli expressa esse cuidado quando recomenda que "para entender a situação atual da pesquisa na área da educação, é preciso recompor sua trajetória, de modo que possamos situar seus avanços e desafios contemporâneos". (ANDRÉ 2006, p. 45). Segundo ela, a origem da pesquisa em educação no Brasil, que inicialmente foi induzida pelos órgãos governamentais com vista a dar subsídios às suas ações no campo da educação escolar e nas políticas educacionais, evolui com a implantação dos cursos de pós-graduação nas décadas de 60 e 70 do século XX e se expande com a criação de grupos de pesquisa fora do espaço da pós-graduação. E nesse momento histórico, ainda que a pesquisa educacional no Brasil seja recente, há um expressivo crescimento e diversificação das pesquisas a partir do qual é possível um olhar crítico sobre seus avanços e suas perspectivas. (ANDRÉ, 2006).

Com esse olhar, Marli André deixou um expressivo legado às diferentes gerações de pesquisadores que buscam fundamentar teórica e metodologicamente sua produção científica. E, para aqueles que tiveram o privilégio de conviver com ela deixou uma lição de vida. Marli mulher, mãe, amiga, professora, pesquisadora, orientadora de tantos pesquisadores que por suas mãos conheceram a beleza e o desafio da produção do conhecimento auscultando a realidade com seus desafios e possibilidades. Sobretudo nos deixou o exemplo da humildade intelectual no enfrentamento dos desafios postos pelas condições objetivas de trabalho, de pesquisa a que todos estamos submetidos. Aprender sempre, buscar compreender sempre, tendo em vista avanços na pesquisa. De uma lição de vida aprendida na e pela prática nasceu uma amizade sólida que ultrapassou o espaço acadêmico.

Neste artigo, para melhor compreender o expressivo legado deixado por Marli selecionamos um conjunto de artigos publicados em diferentes periódicos nacionais para situar, nos caminhos percorridos por André, problemas a serem superados na produção de conhecimentos sobre a pesquisa qualitativa. Procuramos analisar as diferentes problemáticas e os inúmeros desafios que a pesquisadora destacou em sua trajetória, especialmente aqueles decorrentes das dúvidas e soluções identificadas na formação de pesquisadores da área da educação.

\section{Problemáticas e desafios da pesquisa qualitativa em educação}

Para tratar das problemáticas e desafios da pesquisa qualitativa em educação, selecionamos na vasta produção científica de Marli André, sete artigos por ela publicados nas duas primeiras décadas de 2000 e por nós considerados seminais na busca de compreensão do comprometimento da pesquisadora com a qualidade das pesquisas em educação. Buscando compreender os novos rumos das pesquisas em educação, no artigo "Pesquisa em Educação: buscando rigor e qualidade", André (2001) ressalta o crescimento das pesquisas nos últimos vinte anos, na área da pós-graduação brasileira. Período em que ocorreram expressivas mudanças nas temáticas e problemas, nos referenciais teóricos, nas abordagens metodológicas, assim como nos contextos da produção da pós-graduação em educação. De fato, nas décadas de 80 e 90, do século XX, com o crescimento desses cursos, nota-se um redirecionamento das pesquisas em educação, cujos olhares direcionaram-se mais para os processos, do que para os produtos, centrando-se no interior da escola, em 
seus currículos, na sala de aula, na disciplina, na avaliação, priorizando estudos sobre o cotidiano escolar. Questões genéricas são substituídas por questões específicas, localizadas em contextos anteriormente pouco visitados pelos pesquisadores. Tais mudanças mostram que, para além da busca de soluções técnicas, o cenário investigativo abre espaço para problematizações do contexto escolar, para teorias do conflito, para abordagens críticas, recorrendo "não mais exclusivamente à psicologia ou à sociologia, mas à antropologia, à história, à linguística, à filosofia" (ANDRÉ, 2001, p. 53). Mudanças que desde os meados do século XX expressam um tempo marcado por movimentos sociais em defesa da igualdade dos direitos humanos, das causas sociais do período e que resultaram em crescente aumento dos debates na área da educação.

Contrapondo-se à visão positivista de uma postura neutra do pesquisador, as pesquisas em educação passaram a criticar as abordagens economicistas reducionistas que supervalorizavam os dados quantitativos e passando a considerar a íntima relação entre sujeito e objeto, dirigindo um olhar especial às interações entre os componentes do contexto, em especial aos significados atribuídos pelos sujeitos às suas experiências cotidianas.

Tempos em que as temáticas, ao se tornarem mais complexas vão requerer novas abordagens, como os estudos do tipo etnográfico, pesquisa participante, estudo de caso, pesquisa -ação, história de vida, entre outras abordagens metodológicas que nesse período ganharam força na comunidade científica brasileira. Mudanças que provocaram novos questionamentos em relação aos fins da pesquisa e à natureza dos conhecimentos produzidos. Mudanças que também incidem na substituição dos dados resultantes de testes por dados advindos de observações devidamente validadas; na flexibilidade do planejamento da pesquisa; na transparência dos valores e pontos de vista do pesquisador.

Soluções que sinalizam para um maior comprometimento dos pesquisadores com a realidade investigada, ao dar mais visibilidade das necessidades e possibilidades de intervenções no contexto escolar.

E nesse sentido, o artigo "Pesquisas em educação: questões de teoria e método", publicado por André em 2005, discute questões epistemológicas e metodológicas assim como as condições de produção do conhecimento científico nas pesquisas em educação. Suas indagações remetem a pontos nucleares da abordagem qualitativa, entre eles, a questão da natureza dos conhecimentos produzidos, os critérios de avaliação dos métodos científicos, os pressupostos dos métodos e técnicas de avaliação. Mostrando-se preocupada com a qualidade de uma pesquisa, provoca os pesquisadores com novas indagações. O que é uma pesquisa de qualidade? Quem define os critérios para julgar a qualidade de uma pesquisa? Como elaborar instrumentos válidos para avaliar as novas modalidades de pesquisa? Como generalizar resultados?

Dentre os inúmeros questionamentos, Marli tece considerações acerca de possíveis soluções, alertando sobre o risco da diminuição do rigor científico frente ao fascínio da ação. Valendo-se do conceito de professor reflexivo, exemplifica com a pesquisa-ação, as fragilidades desta abordagem quando realizada sem o rigor necessário aos conhecimentos da ação. E nesse sentido, destaca como critérios para julgamento da pesquisa, o reconhecimento externo do projeto de formação (validade externa); os métodos e técnicas utilizados (validação do processo); a consideração dos múltiplos interesses dos participantes (validade democrática); a compreensão da realidade a ser transformada (validade catalítica) e, por último, a busca de diálogo com os pares sobre o problema e resultados da pesquisa (validade dialógica).

É em busca desse rigor na produção de conhecimento que a pesquisadora enfatiza a necessidade de atenção a ser dada à perspectiva do outro, a construção de categorias teoricamente fundamentadas, ao tratamento 
adequado das subjetividades dos atores, além da correta definição de seus papéis e dos critérios a serem adotados na divulgação da pesquisa. Em nome da relevância científica e social da pesquisa em educação, Marli chama a atenção para o processo de transformação de conhecimentos dispersos em conhecimentos sistematizados, para a articulação necessária entre conhecimentos teóricos e conhecimentos da ação. Sinaliza para o processo de decantação das subjetividades dos atores, tendo em vista o reconhecimento e o consenso dos pares, em relação às transformações ocorridas nos conhecimentos anteriores que, ao serem formalizados e legitimados como científicos, tornam-se institucionalizados.

Ao mencionar a importância desse processo que articula saberes da ação com saberes acadêmicos, questiona as condições de produção de novos conhecimentos por discentes, tanto pelo tempo exíguo de realização do mestrado, quanto pela falta de bolsas de estudo a mestrandos e doutorandos sobrecarregados de trabalho em suas instituições e que os impedem de realizar um trabalho com densidade. Também questiona o pouco tempo dos docentes/orientadores para dedicar-se a pesquisa, dada a sobrecarga de trabalho com aulas, comissões, reunião, trabalhos para avaliar etc.

Em 2006, retomando essas mudanças, Marli traça um panorama da jovem pesquisa brasileira, no qual destaca problemas, tanto nos referenciais teóricos quanto nas abordagens metodológicas e nos contextos de produção dos trabalhos científicos. Observa que as preocupações dos pesquisadores ao se voltarem mais para aspectos internos da escola, deslocando-se das questões gerais e externas para questões mais específicas, trazem à tona problemas sobre o uso de novas abordagens, a necessidade de estudos mais críticos que requerem olhar multidimensional buscado em aportes de diferentes campos científicos dentre outros, a sociologia, antropologia, história, filosofia, marcando um momento em que as pesquisas do cotidiano escolar ganham força na comunidade científica da educação brasileira.

Como aspectos problemáticos, o artigo reafirma fragilidades nos contextos de formação de pesquisadores como a falta de apoio das universidades e das agências de fomento às pesquisas; a quase ausência de grupos de pesquisas com um sólido corpo de conhecimentos voltados a constituição de um perfil investigativo próprio ao programa vinculado; a falta de condições dos docentes para a dedicação à pesquisa na universidade. Outros problemas sinalizados pela pesquisadora referem-se à pulverização das temáticas, a fragilidade metodológica, o pouco impacto nas práticas e ainda a preferência dada aos referenciais estrangeiros, a diversidade de citações sem um fio condutor da pesquisa e a falta de clareza em relação ao objeto de estudo. Ainda nesse artigo, a autora advoga a favor de enfrentamento coletivo das questões face ao agravamento das condições de trabalho dos docentes para a conquista da maturidade científica e do devido reconhecimento e respeito da pesquisa educacional. E conclui, perguntando: "Como desenvolver um bom trabalho de pesquisa com pouca disponibilidade de tempo e com um orientador sobrecarregado?" (p.23).

E sua resposta vem imediata, lembrando a tarefa urgente das universidades, dos programas de pós-graduação e dos pesquisadores em lutar pela qualidade das pesquisas e melhores condições de produção de conhecimentos. Bandeira esta, firmemente empunhada por Marli, no limiar do século XXI, em sua brilhante trajetória acadêmica, acentuando um alerta para a busca de maturidade da jovem pesquisa educacional brasileira.

No artigo "Desafios da pós-graduação e da pesquisa sobre a formação de professores", publicado em 2007, com o foco nos cursos de pós-graduação, Marli retoma e amplia problemáticas já tratadas em artigos anteriores, ou seja, a reestruturação dos modelos de formação, novas funções dos grupos de pesquisa e o fortalecimento dos intercâmbios interinstitucionais. Questiona condições da produção de conhecimento e qualidade da pesquisa na área de formação de professores, sobretudo a fragmentação das temáticas, a 
fragilidade das abordagens teórico-metodológicas, reafirmando a importância de um repensar coletivo da melhoria da produção de conhecimentos.

Expondo um breve panorama dos cursos de pós-graduação em educação, mostra seu expressivo aumento no quadriênio entre 1998 a 2002, notadamente a rápida expansão do curso de doutorado nas regiões sul e sudeste. E decorrente desta expansão, destaca um dado novo, ou seja, o perfil dos alunos que procuram o mestrado e doutorado, alunos que já trabalham e buscam uma atualização profissional. Em geral, os que iniciam o mestrado "trazem um repertorio bastante pobre, que é resultante da precariedade de nosso sistema educacional. Apresentam dificuldades de leitura e escrita e desconhecem conceitos básicos da educação" (ANDRÉ, 2007, p. 48). Comenta que grande parte dos que procuram o doutorado mostram falta de domínio da literatura educacional e das habilidades básicas de pesquisa.

Questionando a rígida estrutura dos cursos tradicionais, propõe flexibilização dos esquemas formativos tendo em vista atender a nova realidade dos alunos que, em sua maioria, trabalham e estudam, ofertando disciplinas que atendam suas carências em termos de habilidades para a pesquisa. E indaga se rejeitar a alternativa dos mestrados profissionais seria a melhor solução.

Em relação aos grupos de pesquisa, Marli enfatiza o potencial que apresentam para a melhor qualidade das pesquisas, como espaço que viabiliza discussão coletiva e troca de conhecimentos com pesquisadores mais experientes, além de servir de laboratório de aprendizagem de orientação e avaliação de trabalhos científicos, considerando os doutorandos como futuros orientadores e avaliadores. Ainda, por constituir-se em um espaço favorável para desenvolvimento de um projeto coletivo e consolidação de linhas de pesquisa. Os grupos também se constituem em espaço profícuo para a consecução de projetos interinstitucionais, assim como os de cooperação internacionais, inciativas que abrem oportunidades para apreensão de novas teorias e possibilidades de amplificação e divulgação da produção científica.

Buscando melhor caracterizar problemáticas das pesquisas produzidas nos cursos de pós-graduação em educação, Marli reporta-se a um estudo que analisou 77 trabalhos apresentados nos Endipes ${ }^{1}$ (Encontro Nacional de Didática e Prática de Ensino) entre 1994 e 2000, tendo como objeto o professor pesquisador. E considerou grave um dado que indica que $74 \%$ dos trabalhos não informaram a abordagem metodológica. Dado que Marli considerou uma ausência inaceitável em um trabalho científico. Este e demais dados do trabalho examinado levaram Marli a reafirmar a necessidade de repensar a formação do pesquisador, e questionar as condições de trabalho dos orientadores e as condições de produção do conhecimento científico no Brasil. Amplia o questionamento mencionando a diminuição do apoio financeiro para a pesquisa, a reposição parcial de professores que se aposentam, o aumento do número de alunos que procuram o curso, assim como a falta de recursos para os programas, incluindo o número restrito de bolsas de estudo. Tais fragilidades segundo Marli requer, além de um repensar os cursos de pós-graduação, a melhoria da formação de pesquisadores, uma ação coletiva para melhorar as condições de produção do conhecimento científico.

O artigo "A complexa relação entre pesquisas e políticas públicas no campo da formação de professores", publicado em 2009, expõe a visão abrangente da pesquisadora comprometida com a qualidade das pesquisas, após ter constatado um expressivo aumento dos programas de pós-graduação e das pesquisas na área da

1 Trata-se da tese de Silvana Ventorim : “A Formação do professor pesquisador na produção científica dos encontros de Didática e Prática de Ensino: 1994-2000”, concluída em 2005 no Programa de Doutorado em Educação da Universidade Federal de Minas Gerais.

Em 2008, a pesquisadora já havia tratado dessa temática, no artigo “Grupos de Pesquisa: formação ou burocratização?”, recomendando a troca do papel formalista e burocrático dos grupos de pesquisa por um espaço formativo e de melhoria da qualidade dos cursos de pós-graduação. 
educação. Apontando mudanças ocorridas nos anos 2000, em que num período de cinco anos, o quantitativo de 4.085 dissertações e teses defendidas passou para 8.280, observa que as mudanças também alteraram o foco das pesquisas. Nos anos 1990 o foco era a formação iniciação inicial e nos anos 2000 voltou-se para a profissionalização e a identidade docente. Tais mudanças levaram a pesquisadora a dois importantes questionamentos: um, sobre a queda de pesquisas no âmbito da formação inicial, consideradas pelos órgãos financiadores como pouco rendáveis: "teriam os pesquisadores se curvado às políticas globalizadas? (ANDRÉ, 2009 , p. 271) e outro, "conhecer as opiniões, representações, saberes e práticas do professor, para que?". Para além das descrições e constatações dos discursos docentes, o fundamental seria "compreender o contexto de produção desses discursos e das práticas observadas" (p.272), tendo em vista subsidiar novas práticas e políticas de formação.

Ao priorizar autores estrangeiros, esses estudos apontam a prevalência, na formação, do modelo de racionalidade prática, de acordo com o perfil do professor reflexivo teorizado por Schön, sugerindo um certo modismo. No entanto, a dúvida da pesquisadora é se os pesquisadores têm considerado este conceito na especificidade dos contextos brasileiros. Sobre as metodologias empregadas, a indagação de Marli é: "como superar a profusão de micro estudos, priorizados desde os anos 1990, com a exígua limitação do prazo dado para a elaboração das dissertações?." Uma hipótese seria o fortalecimento dos grupos de pesquisa, transformando-os em espaço potencial de formação do pesquisador.

Para a autora, tem sido muito positivo o uso de novas formas de coleta de dados, como o grupo focal, a vídeo-grafia, o relato autobiográfico. Contudo, os estudos têm mostrado uma certa imprecisão em relação ao objeto de estudo ao tratar da formação de professores. Referindo-se a ênfase na instrumentação quantitativa contemplada pelas políticas neoliberais que supervalorizam "as medidas, os resultados, as evidências" ( $p$. 273) que, ao defender sistemas de avaliação em larga escala, implementam novas formas de regulação e controle dos agentes escolares, professores e alunos.

Marli afirma que apesar do significativo desenvolvimento das abordagens qualitativas nos últimos trinta anos, os estudos quantitativos têm retornado com muita credibilidade. E nesse sentido, as problemáticas até então discutidas pela pesquisadora, em relação à complexidade da área da educação indicam que, quando se trata das relações entre pesquisas da educação e políticas públicas, os artigos reafirmam a necessidade de sistematizações dos conhecimentos docentes acerca dos contextos nos quais estão inseridos.

O artigo "A formação do pesquisador da prática pedagógica", produzido em 2016, aborda o processo levado a efeito na disciplina "Pesquisa e Prática Reflexiva", ministrada no curso de Mestrado Profissional da PUC SP. Partindo da diferença entre a pesquisa acadêmica e a pesquisa da prática pedagógica, tendo em vista dirimir dúvidas dos participantes, destaca a transformação do papel do professor formador que "deixa de ser um transmissor de saberes para ser um profissional prático reflexivo", um profissional "capaz de analisar situações-problema e tomar decisões fundamentadas numa perspectiva teórico-crítica, e em pesquisas que tomam seu campo profissional como objeto de estudo"(ANDRÉ, 2016, p.1).

Considerando que a formação desse profissional vai além de um consumidor ou usuário de pesquisas, o que requer aquisição de habilidades básicas para desenvolver uma atitude de pesquisador, ou seja, escolher um tema, problematizá-lo, formular uma questão central, fazer uma revisão teórica, argumentar, produzir e sistematizar dados, relatar achados. Um rol de conhecimentos que necessita de uma consistente base teórico-metodológica. 
Seguindo a diferenciação entre a pesquisa acadêmica e a pesquisa da prática pedagógica, Marli reconhece a prevalência do caráter teórico da primeira e do caráter prático da segunda, denominada por Gatti (2014) de "pesquisa engajada" e mais centrada na realidade empírica em busca de soluções alternativas para os problemas nela detectados.

Com a experiência adquirida em quase quatro décadas orientando mestrandos e doutorandos, Marli sublinha que não bastava conhecer as ferramentas adequadas para a pesquisa da prática pedagógica, quando se tem em vista uma abordagem qualitativa da pesquisa, mais importante seria saber utilizá-las.

Ponderei a mim mesma que a questão não estaria apenas no quê, nos conteúdos, mas muito mais no como desenvolver os conteúdos. Tinha que orientar a produção individual, mas não podia deixar de promover a discussão coletiva dessas produções. [...]Tinha que respeitar o tempo e o ritmo de cada um, ao mesmo tempo em que estimulava o avanço do grupo" (ANDRÉ, 2016. p. 35. Grifo da autora).

E nessa dinâmica os participantes vão estruturando seus projetos de pesquisa, dirimindo dúvidas sobre seus elementos. Em um trabalho em conjunto com tutores de pequenos grupos, a formação do pesquisador prático possibilitou que os participantes refletissem sobre problemas de suas práticas pedagógicas, aprendendo a problematizá-los, e propor soluções criativas para superá-los. Assim o projeto da pesquisa dos participantes vai sendo elaborado, de forma coerente e teoricamente fundamentado e comprometido em resolver um problema detectado em sua prática pedagógica. Uma construção que aponta não uma distinção senão uma aproximação entre "pesquisa acadêmica" e "pesquisa engajada", como justifica a autora ao afirmar que ambas requerem um rigoroso cuidado metodológico expresso num processo sistemático de planejamento, desenvolvimento e relato de dados. E finaliza a mensagem central da disciplina, lembrando que não existe pesquisa sem rigor teórico-metodológico. 


\section{Considerações finais}

A história da pesquisa qualitativa em educação no Brasil se entrelaça com a trajetória profissional de Marli André, pesquisadora dedicada ao estudo das problemáticas e desafios que permearam e permeiam as pesquisas qualitativas. Para Marli, entender a situação atual da pesquisa na área da educação requer a sua historicização de modo a situar seus avanços e desafios contemporâneos.

Então, para melhor compreender o expressivo legado deixado por Marli André empreendemos um breve percurso na sua trajetória investigativa, o que nos permitiu situar mudanças que marcaram as pesquisas em educação, no Brasil, notadamente a partir dos anos 1960, com o uso da abordagem qualitativa voltada aos estudos do cotidiano escolar. Da mesma forma, apontou para as problemáticas impregnadas nos processos de produção do conhecimento, especialmente aquelas relacionadas às exigências de rigor científico, à desnaturalização da polarização entre método quantitativo e qualitativo, à fundamentação teórica das análises dos dados, ao tratamento das subjetividades e a necessária articulação entre conhecimentos teóricos e conhecimentos da ação.

Nos permitiu, também, entender as implicações das condições objetivas de produção do conhecimento tanto dos pós-graduandos como dos seus orientadores para o desenvolvimento das pesquisas, denunciando a falta de apoio das universidades e das agências de fomento às pesquisas. É marcante sua luta constante e proposição de uma ação coletiva para melhoria dessas condições e essa foi uma bandeira empunhada por Marli em sua brilhante trajetória acadêmica, alertando para a busca de maturidade da jovem pesquisa educacional brasileira.

Reconhecendo a importância da pesquisa qualitativa para a compreensão dos problemas educacionais, Marli André foi uma fiel batalhadora em prol de um rigoroso uso dessa abordagem enquanto valiosa ferramenta para a produção de conhecimentos da realidade escolar. Seus ensinamentos, tão bem mobilizados em cursos de pós-graduação, estão presentes na memória de inúmeras gerações de pesquisadores que a cada dia buscam melhor compreender os contextos escolares nos quais exercem sua atividade profissional. 


\section{REFERÊNCIAS}

ANDRÉ, M. E. D. Pesquisa em Educação: buscando rigor e qualidade. Cadernos de Pesquisa. n. 113, juIho/2001, p. 51-64.

ANDRÉ, M. E. D. Pesquisa em Educação: questões de teoria e método. Educação Tecnológica. Belo Horizonte, v.10, n.1, p. 29-35, jan./jun.2005.

ANDRÉ, M. E. D. A jovem pesquisa educacional brasileira. Diálogo Educacional. Curitiba, n.19, p. 11-24, set./ dez.2006.

ANDRÉ, M.E. D. Desafios da pós-graduação e da pesquisa de formação de professores. Educação \& Linguagem. Ano 10, n. 15, p. 43-59, jan.-jun. 2007.

ANDRÉ, M.E. D. A complexa relação entre pesquisas e políticas públicas no campo da formação de professores. Educação. Porto Alegre, v.32, n.3, p. 270-276, set./dez., 2009.

ANDRÉ, M. E. D. A formação do pesquisador da prática pedagógica. Plurais - revista multidisciplinar. Salvador, v. 1, p. 30-41, jan./abr. 2016.

GATTI, B. A. A Pesquisa em Mestrados Profissionais. Apresentação no / FOMPE - I Fórum de Mestrados Profissionais em Educação. UNEB, Salvador, março, 2014.

MARTINS, P. L. O.; PINTO, N. B.; Problemáticas e desafios da pesquisa qualitativa na educação brasileira sob o inesquecível olhar de Marli André. Formação Docente - Revista Brasileira de Pesquisa sobre Formação de Professores. Belo Horizonte. Vol. 13, no 28 (p. 91-100) 31 dez. 2021. ISSN: 2176-4360. doi https://doi.org/10.31639/rbpfp. v13i28.543 